\title{
Autonome Störungen bei Multipler Sklerose
}

Peter Flachenecker

Neurologische Klinik und Poliklinik der Universität Würzburg

Neurologische Abteilung der Klinik Bavaria Bad Kissingen

psychoneuro 2004; 30 (7): 380-383

Autonome Funktionsstörungen kommen bei rund 80\% der Patienten mit Multipler Sklerose (MS) vor und sind beeinträchtigend. Von besonderer Bedeutung sind Blasenstörungen, nicht nur wegen ihrer potentiellen Auswirkungen auf das Alltagsleben der betroffenen Patienten, sondern auch wegen der Gefahr sekundärer Komplikationen wie häufiger Harnwegsinfekte und fortschreitender Nierenschädigung. In dieser Übersicht werden klinische Manifestationsformen und Häufigkeit der urogenitalen, gastrointestinalen, kardiovaskulären und thermoregulatorischen autonomen Funktionsstörungen bei MS-Patienten besprochen und deren Behandlungsmöglichkeiten aufgezeigt.

$\mathrm{D}$ as autonome Nervensystem ist ein komplexes neurales Netzwerk, das für die Aufrechterhaltung der Homöostase verantwortlich ist und verschiedene Organsysteme reguliert. Kardiovaskuläre, thermoregulatorische, gastrointestinale, urogenitale und andere Funktionen wie die Sekretion exokriner Drüsen oder die Pupillenreaktion stehen unter dem Einfluss des autonomen Nervensystems und sind damit der willkürlichen Kontrolle weitgehend entzogen. Die beiden Schenkel mit gegenläufigen Auswirkungen auf die Effektororgane sind das sympathische und das parasympathische Nervensystem, die sowohl im peripheren als auch im zentralen Nervensystem repräsentiert sind (22). Die multiple Sklerose (MS) als eine Erkrankung des zentralen Nervensystems mit disseminierten, multifokalen Läsionen kann eine Vielzahl von Symptomen verursachen, darunter auch solche des autonomen Nervensystems. Davon sind besonders Störungen von Blasen-, Mastdarm- und Sexualfunktion beeinträchtigend; klinisch weniger bedeutsam sind Störungen der HerzKreislauffunktion sowie Temperaturregulations- und Schweißsekretionsstörungen (Abb. 1).

\section{Blasenfunktion}

Die zwei wesentlichen Funktionen der Blase, die kontinente Urinspeicherung und die periodische, vollständige Blasenentleerung, werden durch zwei getrennte Systeme gewährleistet, die allerdings enge Wechselbeziehungen zur Aufrechterhaltung einer koordinierten Blasenfunktion unterhalten (5). Die Blasenkontinenz während des Speichervorgangs wird durch den $\alpha$-adrenerg (sympathisch) innervierten inneren und den somatisch (N. pudendus) innervierten äußeren Sphinkter gewährleistet. Bei Dehnung der Blasenwand erfolgt die reflektorische Auslösung des Harndrangs und - in Abhängigkeit von der Aktivität übergeordneter Zentren - eine Kontraktion des Detrusor vesicae über den (parasympathischen) N. pelvicus aus dem Sakralmark. Supraspinal wird die Blasenentleerung durch das anatomisch nur unscharf charakterisierte pontine Miktionszentrum gefördert, das wiederum durch zahlreiche $\mathrm{Zu}$ flüsse aus frontalem Cortex, Gyrus cinguli, Lobulus paracentralis und Basalganglien gehemmt wird.

Neurogene Blasenstörungen gehören zu den häufigsten autonomen Symptomen bei der MS. Die Prävalenz beträgt zwischen 50 und $80 \%$. In

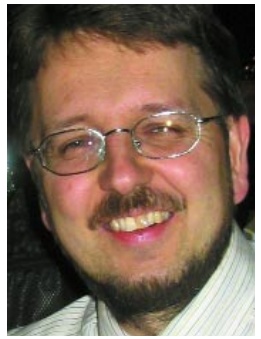

Peter Flachenecker
2\% der Fälle sind sie alleiniges Erstsymptom, und bei immerhin 10-14\% wesentlicher Teil der Erstsymptomatik $(14,15)$. Dabei besteht eine enge Korrelation zwischen dem Schweregrad der Blasenstörung und dem Ausmaß der spastischen Paraparese, was dafür spricht, dass im wesentlichen spinale Läsionen für die Blasenstörung verantwortlich sind (2). Die Patienten beklagen vor allem Symptome einer Detrusor-Hyperreflexie wie Pollakisurie, imperativer Harndrang, Inkontinenz und Enuresis, aber auch einer verzögerten Blasenentleerung wie Starthemmung, Entleerung kleiner Urinportionen, Nachträufeln und Restharnbildung (2). Beide Symptome können auch gleichzeitig auftreten (Tab. 1). Bei 6-30\% der Patienten besteht eine Detrusor-Sphinkter-Dyssynergie (14).

Zur langfristigen Vermeidung sekundärer Komplikationen (rezidivierende Harnwegsinfekte, progrediente Nierenstauung mit der Gefahr des Nierenversagens) ist die frühzeitige Erkennung und symptomorientierte Behandlung von zentraler Bedeutung. Dabei ist oftmals die Bestimmung der Restharnmenge mittels Sonographie oder Einmalkatheter ausreichend. Beträgt die Restharnmenge weniger als $100 \mathrm{ml}$, kann Oxybutinin (Dridase $^{\circledR}$ ) in einer Dosierung von zunächst 2,5-5 mg zweimal täglich und langsamer Steigerung unter regelmäßiger Restharnkontrolle gegeben werden (13). Bei unzureichendem Therapieerfolg (fortbestehende Inkontinenz und Restharnbildung) ist allerdings unbedingt eine urodynamische Untersuchung durchzuführen, um die Speicher- und Entlee- 
rungsfunktion der Blase zuverlässig bestimmen zu können. Bei Blasenentleerungsstörungen mit Restharnbildung kommen $\alpha$-Sympatholytika (Phenoxybenzamin, z.B. Dibenzy$\operatorname{ran}^{\circledR}$ ) oder Parasympathomimetika (Ubretid bzw. Carbachol, z.B. Doryl ${ }^{\circledR}$ ) in Frage (18). In schweren Fällen ist dem intermittierenden Selbst-Katheterismus vor Anlage eines Blasendauerkatheters oder eines suprapubischen Katheters der Vorzug zu geben (1).

\section{Sexualfunktion}

Sympathische Nervenendigungen aus dem thorakolumbalen Grenzstrang und parasympathische Nerven aus dem sakralen Rückenmark formen den Plexus pelvicus, über den die autonome Innervation der Geschlechtsorgane erfolgt. Die Erektion wird durch einen vermehrten Blutfluss infolge einer reduzierten sympathischen ( $\alpha$-adrenerg vermittelten) und einer vermehrten cholinergen Aktivität verursacht und aufrechterhalten, während die Kontraktionen der glatten Muskulatur bei der Ejakulation durch sympathische Aktivität vermittelt werden. In ähnlicher Weise spielen sich diese Vorgänge auch im weiblichen Organismus ab (6).

Über 80\% der männlichen und über 50\% der weiblichen MS-Patienten berichten über Störungen der Sexualfunktion (15), wobei die Häufigkeit der einzelnen Symptome im Zeitverlauf zwar konstant bleibt, aber der Schweregrad der Beeinträchtigungen zunimmt (25). Ganz im Vordergrund der Beschwerden steht bei den Männern die erektile Dysfunktion, während Frauen eine Minderung des sexuellen Verlangens, der Empfindsamkeit und der Orgasmusfähigkeit sowie eine Dyspareunie berichten. Häufig kommen sexuelle Funktionsstörungen gemeinsam mit Blasenstörungen vor und dürften wie diese auf einer spinalen Läsion proximal des Sakralmarks beruhen (3). Auch pontine Läsionen scheinen dafür verantwortlich zu sein, wie eine erst kürzlich publizierte Arbeit mit Hilfe bildgebender Verfahren zeigen konnte (24). Da bei einem Teil der Patienten mit Sexualfunktionsstörung psychische Faktoren zumindest mitbetei-

\section{Abb. 1 Symptome autonomer Funktionsstörungen}

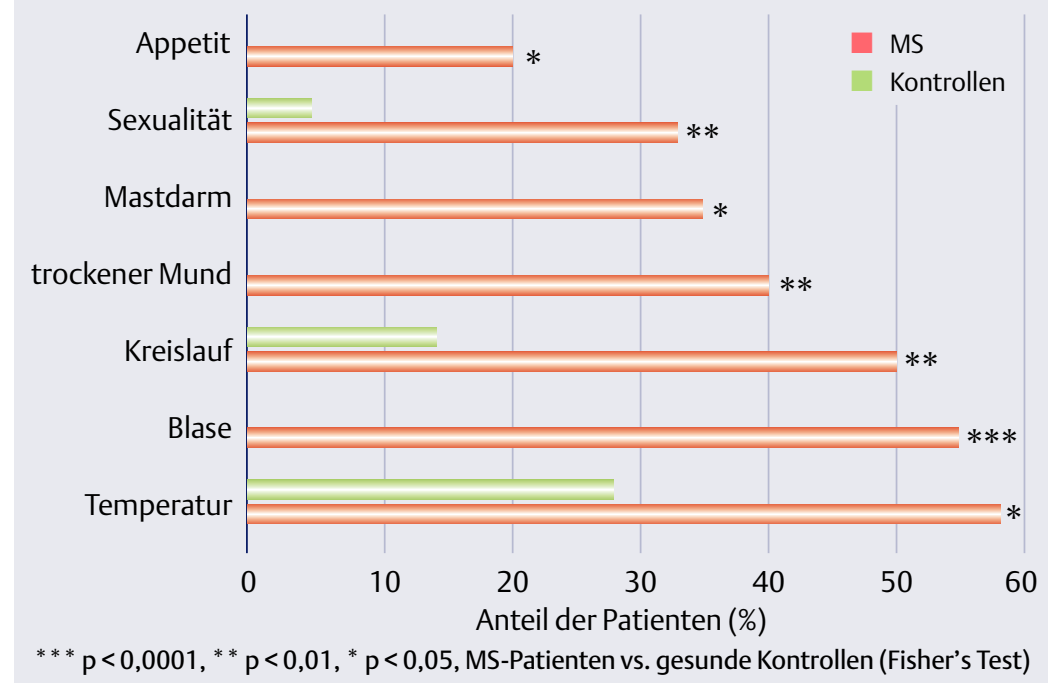

In einer eigenen Untersuchung wurden $\mathbf{4 0}$ MS-Patienten mithilfe eines standardisierten Fragebogens in 13 Kategorien nach Symptomen autonomer Funktionsstörungen befragt und mit 22 gesunden Kontrollpersonen verglichen. Dargestellt sind die Symptome, die bei MS-Patienten signifikant häufiger angegeben wurden

ligt, wenn nicht gar alleinig verantwortlich sind, empfiehlt sich vor allem eine psychologische sexualtherapeutische Beratung. Außerdem können derartige Symptome durch eine Reihe von Medikamenten wie z.B. den Antidepressiva bedingt sein, so dass die kritische Durchsicht der Medikation und ggf. Umstellung bzw. Absetzen überlegt werden sollte. Zur diagnostischen Abklärung der erektilen Impotenz sollte eine urologische Vorstellung mit Messung der nächtlichen Tumeszenz erfolgen (17). Die erektile Dysfunktion kann dann medikamentös mit Sildenafil (Viagra ${ }^{\circledR}$ ) wirksam behandelt werden (Wirksamkeit 60-70\% innerhalb von 3 Wochen). Alternativ können die intrakavernöse Injektion von Papaverin, die intraurethrale Pharmakotherapie mit Alprostadil oder mechanische Hilfen in Form von Vakuumerektionshilfen oder implantierbaren Penisprothesen versucht werden (16).

\section{Gastrointestinale Motilität}

Zur Verdauung, Absorption und dem Transport von Nahrung und Ballaststoffen ist ein abgestimmtes $\mathrm{Zu}$ sammenspiel zwischen Sekretion von Verdauungssäften und Motilität des Magen-Darm-Traktes notwen- dig. Dabei wirkt parasympathische Aktivität (aus dem N. vagus und den sakralen Nerven) motilitätssteigernd, während sympathische Aktivierung die gastrointestinale Motilität hemmt, aber den Muskeltonus der verschiedenen Sphinkteren erhöht. Das intestinale Nervensystem wird von einer Vielzahl von Nervengeflechten innerhalb des Darmes wie dem submukös gelegenen Meissner'schen Plexus, dem myenterischen Auerbach-Plexus, dem tief in der Muskulatur gelegenen Cajal-Plexus und anderen, mukös und submukös gelegenen Plexi gebildet (4).

Gastrointestinale Beschwerden treten bei etwa 40-70\% der Patienten auf (14). Dabei kommen sowohl Obstipation als auch Stuhlinkontinenz vor, häufig auch in Kombination (15). Häufig wird eine Obstipation durch die Immobilität der Patienten bedingt oder zumindest verstärkt. Weitere Manifestationen der gastrointestinalen autonomen Dysfunktion sind Gastroparese mit Völlegefühl, wie sie häufig auch bei Patienten mit diabetischer autonomer Neuropathie gefunden wird, oder Diarrhö (Tab. 1). Die gastrointestinale Motilitätsstörung mit verlängerter Kolon-Transit-Zeit kann durch die Magen-DarmPassage nachgewiesen werden, dane- 


\section{Tab. 1 Symptome} autonomer Funktionsstörungen

\begin{tabular}{|c|c|}
\hline Organsystem & Symptom \\
\hline Herz-Kreislauf & $\begin{array}{l}\text { Orthostatische Intoleranz } \\
\text { - Benommenheit, Schwindel, } \\
\text { Schwäche, Verschwommen- } \\
\text { sehen, Blässe, Angst, Palpita- } \\
\text { tionen, Übelkeit } \\
\text { Störungen des Vasomotoren- } \\
\text { tonus } \\
\text { - Kältegefühl, Blässe, trophi- } \\
\text { sche Störungen }\end{array}$ \\
\hline Schweißdrüsen & $\begin{array}{l}\text { generalisierte oder } \\
\text { lokalisierte Hyperhidrose } \\
\text { Hitzeintoleranz ohne } \\
\text { Schwitzen } \\
\text { mangelndes Schwitzen bei } \\
\text { Hitze oder Fieber }\end{array}$ \\
\hline exokrine Drüsen & $\begin{array}{l}\text { trockene Augen } \\
\text { trockener Mund }\end{array}$ \\
\hline Magen-Darm-Trakt & $\begin{array}{l}\text { Gastroparese } \\
\text { - frühes Sättigungsgefühl, } \\
\text { Völlegefühl, häufige Übel- } \\
\text { keit, Erbrechen unverdauter } \\
\text { Nahrung, Gewichtsverlust, } \\
\text { Anorexie } \\
\text { Diarrhö } \\
\text { Obstipation }\end{array}$ \\
\hline Blase & $\begin{array}{l}\text { imperativer Harndrang } \\
\text { Dranginkontinenz } \\
\text { Harnverhalt }\end{array}$ \\
\hline Genitalorgane & $\begin{array}{l}\text { Erektionsstörungen } \\
\text { Ejakulationsstörungen }\end{array}$ \\
\hline Pupillen & $\begin{array}{l}\text { Akkommodationsstörungen } \\
\text { - Verschwommensehen in der } \\
\text { Nähe oder bei hellem Licht } \\
\text { Nachtblindheit }\end{array}$ \\
\hline
\end{tabular}

ben können endoskopische Verfahren und der Barium-KontrastmittelEinlauf zur Diagnose herangezogen werden. Im klinischen Alltag sind diese Verfahren zumeist jedoch nicht erforderlich. Zur Therapie der Obstipation als der häufigsten gastrointestinalen Dysfunktion kommen peristaltikfördernde Maßnahmen wie ausreichende Bewegung, falls möglich, zumindest aber im Rahmen der physiotherapeutischen Behandlung, Kolon-Massage, ballaststoffreiche Ernährung, Leinsamen und Weizenkleie, osmotisch wirkende Laxantien wie Laktulose oder Macrogol (Movicol ${ }^{\circledR}$ ) und medikamentöse Maßnahmen wie Domperidon (Motilium $^{\circledR}$ ), subkutan appliziertes Prostigmin oder Ceruletid (Takus ${ }^{\circledR}$ ) zum
Einsatz (14). Bei der autonom vermittelten Diarrhö können eine antibiotische Darmsanierung, Adsorbentien wie Kohle-Compretten und motilitätshemmende Substanzen wie Atropin oder Loperamid versucht werden (11).

\section{Schweißsekretion}

Die Schweißdrüsen der Haut werden zwar von postganglionären sympathischen Nervenendigungen innerviert, der Transmitter ist aber im Gegensatz zu den übrigen postganglionären sympathischen Nerven - Azetylcholin. Klinisch manifeste Schweißsekretionsstörungen sind bei der MS selten, auch wenn die sympathische Hautantwort bei über 90\% der Patienten pathologisch ist oder gar fehlen kann (8). Klinisch manifeste Schweißsekretionsstörungen finden sich bei etwa $40 \%$ der Patienten, wobei zumeist die Schweißproduktion an den Füßen beeinträchtigt ist (20). Zur Diagnose dienen der Minor'sche Schweißversuch oder der thermoregulatorische Schweißtest, bei denen die Verteilung der Schweißproduktion mit Hilfe eines Indikators sichtbar gemacht werden kann, der Ninhydrin-Test zur Beurteilung der Schweißproduktion an Händen und Füßen sowie technische Verfahren wie der QSART, bei dem durch Iontophorese mit Azetylcholin die Schweißproduktion über einem bestimmten Hautareal quantifiziert werden kann (11). Im Vordergrund der therapeutischen Bemühungen bei der Hypo- bzw. Anhidrose steht die Vermeidung von Überwärmung, insbesondere, um einen Hitzschlag zu verhüten. Zur Behandlung der Hyperhidrose kann die lokale Applikation von Aluminiumchlorid oder Tannolact versucht werden; in neuerer Zeit steht bei lokalisierter Hyperhidrose mit der subkutanen Injektion von Botulinum-Toxin eine wirksame und gut verträgliche Behandlungsmethode zur Verfügung (19).

\section{Kardiovaskuläre Regulation}

Die sympathische Innervation entstammt dem thorakolumbalen Grenzstrang, vor allem den oberen Abschnitten, während die parasympathische Innervation über den N. vagus erfolgt. Die Afferenzen entsprin- gen den arteriellen Barorezeptoren im Karotissinus, dem Aortenbogen und anderen thorakalen Arterien sowie den Mechanorezeptoren des Herzens und den Dehnungsrezeptoren der Lunge. Aktivierung des sympathischen Nervensystems führt zu einer Erhöhung der Herzfrequenz, der myokardialen Kontraktilität und des peripheren Widerstands, während sich eine gesteigerte parasympathische Aktivität in einer Verringerung von Herzfrequenz und kardialer Pumpfunktion äußert (10).

Das häufigste und die Patienten am meisten belastende Symptom der kardiovaskulären autonomen Dysregulation ist die orthostatische Hypotonie, die bei $24-63 \%$ aller MS-Patienten beobachtet wird (10). Im allgemeinen sind die Patienten hiervon jedoch nur leicht betroffen, allerdings gaben in einer eigenen Untersuchung die MS-Patienten signifikant häufiger an, unter orthostatischen Beschwerden zu leiden, als eine gesunde Kontrollgruppe (50\% vs. $14 \%, \mathrm{p}<0,006$, Abb. 1). Bei der Mehrzahl dieser Patienten traten die Symptome nur gelegentlich auf, und nur zwei der 40 Patienten erlebten derartige Episoden fast immer beim Aufstehen und waren hierdurch beeinträchtigt (12). Allerdings wurde kürzlich auch ein Patient beschrieben, der bereits im Sitzen durch rezidivierende Synkopen beeinträchtigt war und neben einer ausgeprägten orthostatischen Hypotonie Hirnstammzeichen aufwies; kernspintomographisch fanden sich Herde im paramedianen Tegmentum und in der basalen Medulla oblongata. Nach einer KortikosteroidPulstherapie waren sowohl die Hirnstammherde als auch die orthostatischen Beschwerden gebessert, was dafür spricht, dass die orthostatische Intoleranz als Folge der MS anzusehen ist (21).

Als Ursache einer orthostatischen Dysregulation kommen im wesentlichen drei Mechanismen in Frage: (1) eine periphere oder zentrale Sympathikusstörung (hypoadrenerge orthostatische Hypotension), die sich durch einen rasch einsetzenden Blutdruckabfall von mehr als $20 \mathrm{mmHg}$ systolisch innerhalb von drei Minuten auszeichnet, (2) ein übermäßiges venöses Pooling, das ein posturales 
Tachykardiesyndrom mit deutlichem Anstieg der Herzfrequenz (mehr als 30 Schläge pro Minute innerhalb von 10 Minuten) ohne signifikanten Blutdruckabfall bedingen kann, und (3) ein Reflexmechanismus (orthostatische neurokardiogene Synkope), der nach längerem Stehen zur Sympathikusinhibition und vagalen Aktivierung und damit zur Bradykardie und zum Blutdruckabfall führt (7). Tatsächlich ließen sich bei MS-Patienten mit und ohne orthostatische Beschwerden diskrete Veränderungen sowohl bei den autonomen Funktionstests als auch bei der Spektralanalyse der Herzfrequenzvariabilität nachweisen, die in der Zusammenschau auf einen Defekt der sympathischen vasokonstriktorischen Efferenz als Ursache der orthostatischen Intoleranz hindeuten (12).

Eine spezifische Therapie der kardiovaskulären autonomen Dysfunktion ist in den meisten Fällen nicht erforderlich. Die Behandlung der orthostatischen Intoleranz ist nur dann notwendig, wenn sie symptomatisch ist und den Patienten stark belastet oder beeinträchtigt und erfolgt nach den allgemeinen Prinzipien bei der orthostatischen Dysregulation (7). Dafür ist zunächst eine genaue differentialdiagnostische Abklärung mit Messung von Herzfrequenz und Blutdruck im Liegen und Stehen erforderlich, gegebenenfalls unter Einsatz eines Kipptisches. Bei leichter Symptomatik empfehlen sich physikalische Maßnahmen wie reichliche Kochsalzund Flüssigkeitszufuhr, Schlafen in Kopfhochlage, Stehen mit gekreuzten Beinen oder Einnahme einer hockenden Position und Tragen einer Stützstrumpfhose (7). Alle diese Maßnahmen zielen auf eine Verringerung des venösen Poolings. Pharmakologisch kommen bei hypoadrenerger orthostatischer Hypotonie und bei posturalem Tachykardiesyndrom in erster Linie das Mineralokortikoid Fludrocortison (Astonin ${ }^{\circledR}$ ) $(0,1-0,3 \mathrm{mg} / \mathrm{die})$ in Frage. Alternativ steht mit dem $\alpha$-adrenergen Agonisten Midodrin (Gudron ${ }^{\circledR}$ ) ein wirksamer Vasokonstriktor zur Verfügung, der allerdings aufgrund seiner kurzen Halbwertszeit mehrmals täglich eingenommen werden muss
(2,5-5 $\mathrm{mg}$ alle 3-4 Stunden bis maximal $40 \mathrm{mg} /$ die) (9). Demgegenüber konnte die Wirksamkeit der in der Praxis häufig eingesetzten Ergotamin- oder Dihydroergotaminpräparaten in klinischen Studien nicht belegt werden. Als Therapie der Wahl bei Patienten mit nachgewiesener neurokardiogener Synkope gelten $\beta$ Blocker, da sie wahrscheinlich über eine verminderte Ventrikelkontraktibilität eine Überstimulation der Mechanorezeptoren verhindern (23).

Autonomic dysfunction is seen in $80 \%$ of the patients with multiple sclerosis (MS) and may cause clinically significant symptoms in these patients. In particular, bladder disturbances may not only lead to disability but may also be responsible for secondary complications such as urinary tract infections and kidney failure. This reviews deals with the characteristics of urogenital, gastrointestinal, cardiovascular and thermoregulatory autonomic dysfunction in MS patients and discusses treatment options for these often disabling complaints.

\section{Keywords:}

multiple sclerosis - autonomic nervous system - urinary tract disorders - constipation - hypotension

\section{Literatur}

1. Andrews KL, Husmann DA. Bladder dysfunction and management in multiple sclerosis. Mayo Clin Proc 1997; 72: 1176-1183

2. Betts CD, D’Mellow MT, Fowler CJ. Urinary symptoms and the neurological features of bladder dysfunction in multiple sclerosis. J Neurol Neurosurg Psychiatry 1993; 56: 245-250

3. Betts CD, Jones SJ, Fowler CG, Fowler CJ. Erectile dysfunction in multiple sclerosis. Associated neurological and neurophysiological deficits, and treatment of the condition. Brain 1994; 117: 1303-1310

4. Bishop AE, Polak JM. The gut and the autonomic nervous system. In: Mathias C], Bannister R (Hrsg.). Autonomic failure. A textbook of clinical disorders of the autonomic nervous system. 4th Ed. New York: Oxford University Press, 1999: 117-125

5. Bradley WE, Sundin T. The physiology and pharmacology of urinary tract dysfunction. Clin Neuropharmacol 1982; 5: 131-158

6. de Groat WC. Neural control of the urinary bladder and sexual organs. In: Mathias C], Bannister R (Hrsg.). Autonomic failure. A textbook of clinical disorders of the autonomic nervous system. 4th Ed. New York: Oxford University Press, 1999: 151-165

7. DiehI RR, Linden D. Differentialdiagnose der orthostatischen Dysregulationen. Ner- venarzt 1999; 70: 1044-1051

8. Elie B, Louboutin JP. Sympathetic skin response (SSR) is abnormal in multiple sclerosis. Muscle Nerve 1995; 18: 185-189 9. Fealey RD, Robertson D. Management of orthostatic hypotension. In: Low PA (Hrsg.). Clinical autonomic disorders. 2nd Ed. Philadelphia: Lippincott-Raven, 1997: 763-775

10. Flachenecker P. Störungen der HerzKreislauf-Funktion und des Schlafes bei der Multiplen Sklerose. In: Zettl UK, Mix E (Hrsg.). Multiple Sklerose. Kausalorientierte, symptomatische und rehabilitative Therapie. Heidelberg: Springer-Verlag, 2001: 215-234

11. Flachenecker P. Störungen des autonomen Nervensystems. In: Dodel R, Klockgether T (Hrsg.). Lehrbuch Neurologie. Wissenschaftliche Verlagsgesellschaft, 2004 (im Druck)

12. Flachenecker $P$, Wolf $A$, Krauser M, Hartung HP, Reiners K. Cardiovascular autonomic dysfunction in multiple sclerosis: correlation with orthostatic intolerance. J Neurol 1999; 246: 578-586

13. Fowler C]. Investigation of the neurogenic bladder. J Neurol Neurosurg Psychiatry 1996; 60: 6-13

14. Frontoni M, Giubilei F. Autonomic dysfunction in MS. Int MS J 1999; 6: 79-87

15. Hennessey A, Robertson NP, Swingler R, Compston DA. Urinary, faecal and sexual dysfunction in patients with multiple sclerosis. J Neurol 1999; 246: 1027-1032

16. Hoffmann F. Symptomatische Therapie. In: Schmidt RM, Hoffmann F (Hrsg.). Multiple Sklerose. 3rd Ed. München: Urban \& Fischer, 2002: 182-236

17. Kirkeby HJ, Poulsen ZV, Petersen T et al. Erectile dysfuntion in MS. Neurology 1988; 38: 1366-1371

18. Mitchell G. Update on multiple sclerosis therapy. Med Clin North Am 1993; 77: 231-249

19. Naumann M, Flachenecker P, Bröcker EB, Toyka KV, Reiners K. Botulinum toxin for palmar hyperhidrosis. Lancet 1997; 349: 252

20. Noronha MJ, Vas CJ, Aziz H. Autonomic dysfunction (sweating responses) in multiple sclerosis. J Neurol Neurosurg Psychiatry 1968; 31: 19-22

21. Sakakibara R, Mori M, Fukutake T, Kita $\mathrm{K}$, Hattori T. Orthostatic hypotension in a case with multiple sclerosis. Clin Auton Res 1997; 7: 163-165

22. Shields RW. Functional anatomy of the autonomic nervous system. J Clin Neurophysiol 1993; 10: 2-13

23. Sra JS, Jazayeri MR, Avitall B et al. Comparison of cardiac pacing with drug therapy in the treatment of neurocardiogenic (vasovagal) syncope with bradycardia or asystole. N Engl J Med 1993; 328: 1085-1090

24. Zivadinov R, Zorzon M, Locatelli L et al. Sexual dysfunction in multiple sclerosis: a MRI, neurophysiological and urodynamic study. J Neurol Sci 2003; 210: 73-76

25. Zorzon M, Zivadinov R, Monti Bragadin $L$ et al. Sexual dysfunction in multiple sclerosis: a 2-year follow-up study. J Neurol Sci 2001; 187: 1-5

\section{Korrespondenzadresse:}

Dr. med. P. Flachenecker

Abteilung für Neurologie, Klinik Bavaria

Von-der-Tann-Str. 18-22

D-97688 Bad Kissingen

E-Mail: peter.flachenecker@surfeu.de 